ISSN 0001-6002/2001/43/4/178-181

Acta Médica Costarricense,(C2001

Colegio de Médicos y Cirujanos

Caso Clínico

\title{
Abdomen agudo y embarazo: Placenta Percreta
}

\author{
Horacio Massotto-Chaves, ${ }^{1}$ Roy Wong Mc Clure ${ }^{2}$
}

\begin{abstract}
Resumen: La ruptura uterina espontánea por placenta percreta, sin historia de trauma o infección, es una patología extremadamente rara y responsable de una alta morbimortalidad materna. El diagnóstico prenatal de placenta percreta es importante para evitar resultados catastróficos debido a este desorden obstétrico, y diversos procedimientos son utilizados para lograr esta meta. Se presenta un caso de placenta percreta, con búsqueda de expedientes clínicos por esta patología, en el Hospital Monseñor Sanabria, desde 1994 hasta 1999, y con revisión de la bibliografía.
\end{abstract}

Descriptores: Hemorragia obstétrica, placenta percreta, embarazo.

Recibido: 26 de febrero de 2001.

Aceptado: 14 de agosto de 2001.

\section{Presentación del caso}

Paciente femenina, 26 años, gesta 3, para 2, abortos 0, cesáreas 0 , con antecedente de legrado uterino por retención de restos placentarios. Se presenta con edad gestacional por fecha de última regla de 28,4/7 semanas, con primer control prenatal a las 11 semanas de gestación y cuatro controles subsecuentes documentados en el expediente clínico; todos con parámetros dentro de los límites normales, sin ultrasonido registrado. Previo buen estado general, la paciente presenta cuadro caracterizado por dolor abdominal de inicio súbito sin antecedente de trauma, de localización difusa que luego se focaliza en hipogastrio, de moderada a severa intensidad, fijo, sin irradiación, sin alivio espontáneo, asociado a vómitos número uno de contenido alimentario, persistiendo con dolor ya caracterizado, durante 14 horas.

La paciente es valorada en la Clínica de Paquera, Puntarenas, donde se documenta una PA de 110/60mm Hg., una FC de 100 lat/min, está afebril, alerta, activa, consciente, orientada, pálida, con fases de dolor, quejumbrosa. Al examen físico el abdomen presenta distensión, la auscultación con peristalsis intestinal disminuida, hay dolor importante difuso a la palpación del abdomen y signo de Blumberg positivo, con defensa de la pared abdominal no voluntaria, la frecuencia cardiaca

Abreviaturas: PA, presión arterial; FCF, frecuencia cardiaca fetal; cpk, creatin cinasa; AFPM, alfa feto proteína materna; FC, frecuencia cardiaca.

Médico Asistente Cirugía General, Hospital Monseñor Sanabria, Puntarenas, C.C.S.S.

2 Médico Asistente General. Clínica de Paquera y Cóbano, Puntarenas, C.C.S.S.

Correspondencia: Roy Wong Mc Clure. Apdo. 4060-1000 San José, Costa Rica. Correo electrónico: roywcr@hotmail.com fetal (FCF) es de 152 lat/min.; al tacto vaginal el cuello uterino está cerrado, formado, sin evidencia de sangrado transvaginal o hidrorrea en la especuloscopía.

La paciente se refiere al Servicio de Emergencias del Hospital Monseñor Sanabria, con impresión diagnóstica de: Embarazo 28, 4/7 semanas, dolor abdominal en estudio, a descartar abdomen agudo.

En este centro hospitalario se encuentra una paciente pálida, criodiaforética con PA al ingreso de 100/60 mmHg. y FC de 79 lat/min, FCF de 140 lat $/ \mathrm{min}$. Tres horas después la PA está en 88/51 mmHg., la FC en 124 lat/min. y la FCF es de 100 lat/min.; es valorada por el Servicio de Cirugía General y Obstetricia, y se decide realizar laparotomía exploratoria de urgencia, por compromiso del estado hemodinámico de la paciente y su crítica condición. No se contaba en ese momento con radiólogo para efectuar ultrasonido. Se aplicaron 12 mg de dexametasona i.v. Se realizó una laparotomía exploratoria, hallándose una perforación del fondo uterino por placenta percreta, con $3000 \mathrm{~mL}$. de sangre libre en la cavidad peritoneal. No había invasión de órganos intraabdominales por el tejido placentario. Se practicó cesárea y se concluyó el procedimiento realizando una histerectomía con preservación de los ovarios. Se administraron $3700 \mathrm{~mL}$. de cristaloides y $1500 \mathrm{~mL}$. de glóbulos rojos empacados durante el transoperatorio. La paciente tuvo una buena evolución postoperatoria egresando al quinto día del hospital.

Se obtuvo un producto masculino, de 1100 gramos con Apgar de 1 al minuto, 1 a los cinco minutos, 4 a los diez minutos y 6 a los quince minutos, pero que falleció a los 25 días en el Hospital Nacional de Niños, con sepsis por cándida, quiste panencefálico e hipoplasia pulmonar derecha. El diagnóstico histopatológico de la pieza quirúrgica es de placenta percreta total en el fondo uterino. 
Por medio del sistema automatizado de registros médicos se realizó una investigación en el Hospital Monseñor Sanabria y se revisaron los expedientes clínicos, con posible placenta acreta según la Clasificación Internacional de Enfermedades (CIE 9 y 10), desde 1994 hasta 1999, pero no se documentó ningún otro caso de acretismo placentario en ninguna de sus formas.

\section{Discusión}

La placenta percreta corresponde a una anormalidad de penetración dentro de la pared uterina, y es la más severa dentro de este tipo de patologías. ${ }^{1-3}$ La invasión de las vellosidades coriónicas solo hasta el miometrio se denomina plancenta acreta, y cuando las vellosidades coriónicas invaden el miometrio hasta de abajo de la serosa uterina, se llama placenta increta. Se llama placenta percreta cuando el tejido trofoblástico atraviesa completamente la pared uterina. ${ }^{4,5}$

El acretismo placentario en todas sus formas presenta una incidencia de 1 en cada 93000 embarazos, siendo la placenta percreta una anomalía extremadamente rara, la cual se documenta entre el 5 y el 7\% del total de placentas acretas; correspondiendo a una incidencia, mundialmente, de 1 placenta percreta por cada 140.000 embarazos. ${ }^{2,6,7}$

Esta anormalidad en la adhesión placentaria es causada por la ausencia o deficiencia de la membrana de Nitabuch, que es una capa fibrinoide que separa al corion frondoso de la decidua basal y en el parto forma la membrana de separación entre la placenta y el útero, lo cual evita que la placenta pueda infiltrar el miometrio y dar lugar a esta entidad patológica. Irvin y Hertin postularon, en 1937, que la placenta acreta es causada por una deficiencia en la capa decidual, sin embargo, no se conocen los factores que predisponen a esta patología. ${ }^{1}$

A pesar de ser de etiología desconocida, la bibliografía evidencia que tienen alto riesgo aquellas mujeres multíparas, con cesáreas anteriores y con placenta previa, así como también se describe que el trauma del endometrio, ya sea por cesárea, exploración uterina por productos de la concepción retenidos, o por curetaje, predispone a la paciente a implantación anómala en embarazos subsecuentes. ${ }^{1,2,7,8}$

Las pacientes con cicatriz uterina por diversos procedimientos médicos cuentan con un riesgo 5 veces mayor ${ }^{1}$ de presentar placenta de implantación baja, en cuanto a la población en general, y por ende mayor riesgo de acretismo placentario. Clark y colaboradores ${ }^{9}$ encontraron que el riesgo de placenta acreta era del $5 \%$ en pacientes con placenta previa y con útero sin cicatrices, pero éste se incrementa en un $67 \%$ en pacientes con 4 o más cesáreas previas.

El diagnóstico de placenta percreta es usualmente clínico. Puede manifestarse como hemoperitoneo durante el periodo anteparto, al presentarse ruptura uterina consecuente con la protrusión del miometrio por las vellosidades coriónicas, esto ocurre típicamente en el segundo trimestre del embarazo, pero se ha informado tan temprano como en la 12a. semana de gestación. La invasión placentaria del miometrio es un proceso indoloro y se manifiesta clínicamente hasta que la pared uterina se rompe o perfora. La presentación inicial puede incluir shock hipovolémico, dolor abdominal agudo o muerte fetal. ${ }^{2}$ La confirmación histopatológica documentada de la implantación anómala es deseable, pero frecuentemente no obtenible.

La detección precoz, previa al parto es de relevante importancia, ya que con ello se puede realizar una cirugía electiva con menor riesgo de hemorragia catastrófica y, además, promover la maduración pulmonar del producto.

En la última década se ha utilizado el ultrasonido para la detección prenatal de esta anomalía placentaria, ${ }^{7}$ sin embargo, el método, según describen Price y colaboradores, ${ }^{1}$ es de un alto grado de exactitud para diagnosticar la placenta previa, pero no para el diagnóstico de placenta percreta, criterio que contradice lo documentado por Ahmed y Chilton en 1996. ${ }^{10}$ No obstante, actualmente la bibliografía expone la utilidad de las imágenes por resonancia magnética (MRI) en la detección prenatal de placenta percreta, sin poderse documentar aún la sensibilidad y especificidad del procedimiento, por la limitada experiencia existente, y se recomienda la utilización de la MRI solo en aquellos casos en los que existan dudas en cuanto a la valoración ultrasonográfica, por posibles efectos deletéreos desconocidos y por el costo de la misma. ${ }^{11,12} \mathrm{Se}$ describe la utilización de la angiografía ultrasónica con amplitud de potencia, con criterios establecidos para detectar la progresión de la hipervascularidad úteroplacentaria anormal en la placenta acreta, método que supera la detección con doppler convencional. Rara vez la placenta percreta es diagnosticada preoperatoriamente, pero puede producir síntomas y signos de invasión de estructuras adyacentes que orienten al diagnóstico. ${ }^{1,4,13}$

Meehan y colaboradores ${ }^{14}$ utilizaron como medida de sospecha diagnóstica de placenta percreta, la presencia de hematuria, la cual solo se manifiesta cuando la entidad patológica invade la vejiga urinaria y se encuentra en implantación placentaria baja, ya que cuando es de implantación alta la hemorragia intraabdominal es la principal evidencia. ${ }^{15}$ Con base en esta señal, Taefi y colaboradore ${ }^{16}$ describieron un caso de seis cesáreas anteriores con hematuria, el cual tuvo evaluación cistoscópica y biopsia, obteniendo como complicación una hemorragia profusa que requirió histerectomía y resección de la porción de la vejiga, para control del sangrado. Estos autores recomiendan utilizar la cistoscopía solo para la evaluación de las pacientes con riesgo de placenta percreta y la presencia de hematuria, sin la realización de biopsia, ya que se considera contraindicada. ${ }^{10,16}$

Como parte de los intentos por el diagnóstico precoz de placenta percreta, se ha puesto en práctica la determinación de creatin cinasa (CPK), en las madres con elementos clínicos o ultrasonográficos sospechosos de esta anomalía. La determinación de CPK se realiza porque la invasión trofoblástica del 
miometrio produce elevación de la misma en la circulación materna, debido al daño celular uterino. También se ha documentado la elevación de la alfa feto proteína materna (AFPM) en el acretismo placentario, la cual no es patognomónica de esta entidad, pero puede ser un elemento más para sustentar el diagnóstico. Por lo tanto, se sugiere que una elevación inexplicada de CPK o de AFPM, con hallazgos sugestivos de placentación anómala, ya sean clínicos, ultrasonográficos o de MRI debe de alertar de esta posible patología. ${ }^{4,17-19}$

El diagnóstico diferencial de placenta percreta, se debe realizar con todas aquellas entidades que puedan llegar a producir una ruptura espontánea del útero, tal como multiparidad con formación previa de tejido fibroso y violenta reacción uterina, anormalidad uterina, labor precipitada, labor obstruida resaltando macrosomía, mala presentación o pelvis contraída, entre otros. ${ }^{6}$

El manejo de la placenta percreta requiere, por lo general, de extirpación quirúrgica de todos los tejidos involucrados por el tejido trofoblástico invasor, con manejo agresivo del volumen intravascular y cirugía reconstructiva en casos necesarios. El éxito del tratamiento consiste en el control de la hemorragia, que por lo general requiere de histerectomía abdominal total $\mathrm{y}$ en ocasiones subtotal. ${ }^{2,20}$

El manejo conservador, tal como la ligadura de las arterias hipogástricas o uterinas, no es usualmente exitoso, dando como resultado la prolongación del tiempo operatorio y, además, el cierre del acceso para realizar técnicas como la arteriografía. ${ }^{1,21}$ La embolización por medios angiográficos de las arterias ilíacas internas, ha evidenciado resultados satisfactorios y con menores pérdidas sanguíneas, destacando con esto la importancia del manejo multidisciplinario, con la participación del radiólogo intervensionista, desde el inicio hasta el fin del procedimiento quirúrgico..$^{5,21,22}$

La terapia conservadora para preservar el útero y por ende la fertilidad, se ha indicado solo en aquellas situaciones en las que se presente placenta acreta. Se han intentado diversos procedimientos tales como la administración de prostaglandina $\mathrm{F} 2 \mu$, norepinefrina, cloruro de potasio o arginina vasopresina, para mantener el tono miometrial y el control del sangrado. Sin embargo, esto no ha sido efectivo en la mayoría de los casos, por la pérdida uterina de reactividad vasoconstrictora, ya que se presenta depresión o bloqueo de la misma23 y solo la administración de vasopresina se describe en un estudio como efectiva. ${ }^{24} \mathrm{El}$ empacamiento uterino como otra medida conservadora también ha brindado buenos resultados. , $22,25,26^{2}$

Las complicaciones de la placenta percreta se pueden dividir en complicaciones preoperatorias y postoperatorias, en el primer grupo tenemos la invasión de estructuras vecinas como la vejiga urinaria, con altos índices de mortalidad materna, la cual oscila entre el $3.3 \%$ y el $9.5 \% 2$ y fetal/neonatal, inestabilidad hemodinámica con shock hipovolémico y la muerte. Se pueden enlistar en el segundo grupo: las coagulopatías, la formación de fístulas, la estenosis uretral, la formación de litos, el compromiso renal, los abscesos pélvicos y renales y la hemorragia tardía. ${ }^{1}$

La placenta percreta es, en conclusión, una anormalidad obstétrica rara, de etiología desconocida, donde el diagnóstico prenatal es lo óptimo, dada su alta morbimortalidad, pero no es el objetivo más sencillo de alcanzar. Debe de presentarse como diagnóstico diferencial ante la sospecha de abdomen agudo y embarazo, recordando siempre que "el rápido diagnóstico, reemplazo de productos sanguíneos y laparotomía, son los pasos claves para el manejo exitoso de esta condición de emergencia". ${ }^{6}$

\section{Agradecimientos}

Nuestro especial agradecimiento a los Servicios de Estadística y Archivo del Hospital Monseñor Sanabria y Clínica de Paquera por el apoyo incondicional brindado.

\section{Abstract}

Spontaneous uterine rupture, without a history of trauma or associated infection by placenta percreta is an extremely rare pathology and it's responsible for high maternal morbidity and mortality. The prenatal diagnosis of placenta percreta is important to avoid catastrophic results after the obstetrical disorder and different procedures are used to get to this goal. We present here a case of placenta percreta and a revision of cases with this diagnosis from 1994 to 1999 at the Monseñor Sanabria Hospital, and a review of the literature.

Key words: Obstetric Hemorrhage, placenta percreta, pregnancy.

\section{Referencias}

1. Price F, Resnik E, Heller K, Christopherson W. Placenta previa percreta involving the urinary bladder: A report of two cases and review of the literature. Obstet Gynecol 1991: 38(3): 508-511.

2. Smith L, Mueller P. Abdominal pain and hemoperitoneum in the gravid patient: A case report of placenta percreta. Am J Emerg Med 1996; 14(1): 45-47.

3. Pearigen PD. Unusual causes of abdominal pain. Em Med Clin NA 1996; 14(3): 593-613.

4. Ophir E, Tendler R., Odeh M., Khouri S., Oettinger M. Creatine Kinase as a biochemical marker in diagnosis of placenta increta and percreta. Am J Obstet Gynecol 1999; 180(1): 1039-1040.

5. Dubois J, Garel L, Grignon A, Lemay M, Leduc L. Placenta percreta: Balloon occlusion and embolization of the internal iliac arteries to reduce intraoperative blood losses. Am J Osbtet Gynecol 1997; 176(3): 723-726.

6. Imseis H, Murtha A, Alexander K, Barnett B. Spontaneous Rupture of Primigravid Uterus Secondary to Placenta Percreta. J Reprod Med 1998;43(3): 233-236. 
7. Litwin MS, Looughlin KR, Benson CB, Droege GF, Richie JP. Placenta percreta invading the urnary bladder. Br J Urol 1989; 64: 283-6.

8. Klotz PG, Khalaff HM. Placenta percreta invading the bladder: Report of 2 cases. J Urol 1989; 141: 938-9.

9. Clark S, Koonings P, Phelan J. Placenta previa/accreta and prior cesarean section. Obstet Gynecol 1985;66(1): 89-92.

10. Ahmed T, Chilton C. Case Report:Placenta percreta involving urinary bladder. Br. J.Urol 1996;78: 140.

11. Thorp JM, Councell Rb, sandridge DA, Wiest HH. Antepartum diagnosis of placenta previa by magnetic resonance imaginig.Obstet Gynecol 1992;80(3): 506-8.

12. Kay HH, Spritzer CE. Preliminary experience with magnetic resonance imaging in patients with third-trimester bleeding. Obstet Gynecol 1991;78(3): 424-9.

13. Chou M., Shih E. Prenatal diagnosis of placenta previa accreta with power amplitude ultrasonic angiography. Am J Obstet Gynecol 1997; 177(6): 1523-1525.

14. Meehan F, Casey C, Costello J, Connolly C. Placenta previa percreta with bladder involvement. Obstet Gynecol Surv 1989; 44: 835-40.

15. Gribble RK, Fitzsimmons JM. Placenta previa percreta with fetal survival. Am J Obstet Gynecol 1985;153(3): 314-6.

16. Taefi P, Kiser T, Sheffer J, Courey N, Hodson J. Placenta percreta with bladder invasion ahd massive hemorrhage. Obstet Gynecol 1970; 36(5): 686-7.

17. Kupferminc M, Tamura R, Wigton T, et al. Placenta accreta is associated with elevated maternal serum alphafetoprotein. Obstet Gynecol 1993; 82(2): 266-269.

18. Zelop C, Nadel A, Figoletto F et al.: Placenta accreta /percreta/increta: A cause of elevated maternal serum alphafetoprotein. Obstet Gynecol 1992; 80(4): 693-694.

19. Robinson L, Grau P, Crandall BF. Pregnancy outcomes after increasing maternal serum alpha-fetoprotein levels. Obstet Gynecol 1989; 74(1): $17-20$.

20. Clark SL, Yeh S, Phelan JP, Bruce S, Paul RH. Emergency hysterectomy for obstetric hemorrhage. Obstet Gynecol 1984;64(3): 376-80.

21. Mitty HA, Sterling KM, Alvarez M, Gendler R. Obstetric hemorrhage: prophylactic and emergency arterial catheterization and embolotherapy. Radiology 1993;188(1): 183-7.

22. Nelson S, Suresh M. Lack of reactivity of uterine arteries from patients with obstetric hemorhage. Am J Obstet Gynecol 1992;166(5): 14361443.

23. Greenwood LH, Glickman MG,Schwartz PE, Morse SS, Denny DF. Obstetric and nonmalignant gynecologic bleeding: treatment with angiografic embolization. Radiology 1987; 164(1): 155-9.

24. Lurie S, Appelman Z, Katz Z. Intractable postpartum bleedinig due to placenta accreta: local vasopresssin may save the uterus. Br J Obstet Gynecol 1996; 103: 1164.

25. Druzin ML. Packing of lower uterine segment for control of postcesarean bleeding in instances of placenta previa. Surg Gynecol Obstet 1989; 169: 543-5.

26. Moise KT, Belfort MA. Damage control for the obstetrical patient. Surg Clin NA 1997; 77(4): 835-52. 